\title{
Vorstellung der Hippokratischen Gesellschaft Schweiz
}

\author{
Im Namen der Hippokratischen Gesellschaft Schweiz: Raimund Klesse ${ }^{a}$, Susanne Lippmann-Rieder \\ ${ }^{a}$ Dr. med., Präsident, Mitglied der FMH \\ ${ }^{b}$ Dr. med., Vizepräsidentin, Mitglied der FMH
}

Die Hippokratische Gesellschaft Schweiz besteht seit 1999 und ist eine gesamtschweizerische Ärztevereinigung. Unsere Gesellschaft befasst sich mit Fragen der ärztlichen Ethik und der medizinischen Ausbildung sowie mit gesundheits- und standespolitischen Themen. Interessierte Mitglieder und Freunde ${ }^{1}$ unserer Gesellschaft pflegen regelmässig den Austausch über diese Themen und über allgemeine Fragen, die in der täglichen Arbeit in Praxis oder Spital auftreten. Daraus resultieren Publikationen, die Grundlagen für Vernehmlassungsantworten und Anregungen für Vorträge und Veranstaltungen unserer Gesellschaft sind.

\section{Wofür setzt sich die Hippokratische Gesellschaft Schweiz ein?}

Die Hippokratische Gesellschaft Schweiz fördert eine Medizin der Menschlichkeit, basierend auf einem personalen Menschenbild und der vertrauensvollen Arzt-Patient-Beziehung. Grundlage sind die im Hippokratischen Eid zum Ausdruck gebrachten Werte, insbesondere die Ehrfurcht vor dem Leben, wie sie auch

\section{Die medizinische Forschung muss sich aus einer fehlgeleiteten und schädlichen Profitorientierung befreien.}

Albert Schweitzer beschrieben hat [1]. Angesichts fataler Fehlentwicklungen im Gesundheitswesen, wie der Missachtung der Unantastbarkeit menschlichen Lebens vom Lebensanfang bis zum Lebensende, der Eugenik, der Ökonomisierung und der Einführung einer Zweiklassenmedizin, setzen wir uns dafür ein, den hippokratischen Grundsätzen des Arztberufes wieder mehr Gewicht zu verleihen. Der Verein setzt dabei in Anlehnung an den Weltärztebund das Wohl des Patienten an erste Stelle, unabhängig von Rasse, Hautfarbe, Geschlecht, Sprache, Religion, politischer und sonstiger Überzeugung, nationaler oder sozialer Herkunft, Eigentum, Geburt oder sonstigen Umständen.

Anlass für die Gründung unserer Gesellschaft waren die politischen Bestrebungen zur Strafbefreiung der aktiven Patiententötung in der Schweiz. Dem traten wir als Ärzte entschlossen entgegen. Im interdisziplinären Dossier zur «Legalisierung der 'aktiven Sterbehilfe' in der Schweiz?» [2] begründeten wir zuhanden des Bundesrates, der Parlamentarier und der interessierten Öffentlichkeit ein klares Nein in dieser Frage. Die anthropologische Gegebenheit, dass der Mensch als soziales Wesen während der ganzen Lebensspanne in unterschiedlicher Ausprägung auf Bindung und Beziehung zu seinen Mitmenschen angewiesen ist, bedeutet, dass Autonomie ohne Verbundenheit nicht möglich ist. Die Beziehung zwischen Arzt, Angehörigen, anderen Mitmenschen und dem Kranken trägt somit wesentlich zu der Einstellung des Kranken gegenüber seinem Leben und Leiden bei. Dies betrifft alle Lebensfragen, so auch die Frage der Suizidalität. In der Sozialnatur begründet sich auch die Fürsorge- und Garantenpflicht des Arztes gegenüber seinen Patienten, die nicht im Widerspruch steht zur informierten, gleichwertigen Arzt-Patient-Beziehung. Dem Patienten durch explizite "Nichteinmischung» eine falsch verstandene Autonomie zuzuschreiben heisst letztendlich, ihn menschlich und medizinisch im Stich zu lassen.

\section{Heilende Medizin sieht den Menschen ganzheitlich}

Die Hippokratische Gesellschaft Schweiz wehrt sich gegen die Herabwürdigung des Patienten zum Kunden und des Arztes zum Leistungserbringer. Eine heilende, menschlich und fachlich gute Medizin entsteht auf dem Boden einer ganzheitlichen, anthropologisch begründeten Auffassung vom Menschen. Sie lässt dem Patienten das zukommen, was er zu seiner Gesundung tatsächlich braucht. Eine solche Haltung ist weder mit einer utilitaristisch begründeten Mangelversorgung noch mit einer ökonomistisch-gewinnorientierten Herangehensweise, die am Patienten vorbei in einen Mangel oder ein Zuviel münden kann, vereinbar. Die medizinische Forschung muss sich aus einer fehlgeleiteten und schädlichen Profitorientierung be- 


\section{Positionsbestimmung der Hippokratischen Gesellschaft Schweiz}

Folgende Grundsätze und Überzeugungen leiten unsere Aktivitäten und Stellungnahmen

Achtung vor dem Leben:

Das Lebensrecht, wie es die allgemeine Erklärung der Menschenrechte jedem garantiert, weil er Mensch ist, ist die Grundlage des menschlichen Zusammenlebens zu allen Zeiten und allen Orten in Sicherheit und Würde. Der Schutz des Lebens ist grundlegender Bestandteil der ärztlichen Ethik und ureigene Aufgabe des Arztes. Die Hippokratische Gesellschaft Schweiz wendet sich daher entschieden gegen jede Legalisierung von Patiententötungen.

Heilen und Lindern:

Es ist Aufgabe des Arztes, nach bestem Wissen und Gewissen Krankheiten zu heilen, Leiden zu lindern und den Patienten im Verlauf seiner Erkrankung zu begleiten. Jeder Patient soll die bestmögliche, seiner individuellen Situation angemessene medizinischeTherapie erhalten. Die Hippokratische Gesellschaft Schweiz fördert daher eine qualitativ hochstehende medizinische Ausbildung und Behandlung. Auch unterstützt sie den Ausbau der Palliativmedizin und -pflege.

Die Vertrauensbeziehung zwischen Arzt und Patient ist entscheidend für die ärztliche Tätigkeit. Die Gewissheit, sicher aufgehoben zu sein, entsteht, wenn der Arzt sich vorbehaltlos und uneigennützig für seine Patienten einsetzt (Garantenstellung) und die Beziehung durch das Arztgeheimnis geschützt ist. Dazu gehören die freie Arztwahl, die sorgfältige Wahrung des Datenschutzes und die Pflege eines vertrauenerweckenden Arztbildes in der Öffentlichkeit.

Schutz der alten und kranken Menschen:

Das Gesundheitswesen soll auf den Prinzipien der Solidarität und Subsidiarität basieren. Die ethische Durchbildung einer Gesellschaft zeigt sich darin, wie sie mit alten, kranken und behinderten Menschen umgeht. Nur wenn auch für die Schwächsten gesorgt ist, kann sich jeder Mensch sicher fühlen. Entscheidungen im Gesundheitswesen müssen sich an ethischen und wissenschaftlichen Grundsätzen orientieren und dürfen nicht in erster Linie von ökonomischen Gesichtspunkten geleitet sein. Durch sorgfältige und richtige Prioritätensetzung und gemeinsame Anstrengung aller gesellschaftlich Beteiligten kann eine gute Gesundheitsversorgung auch in einer finanziell angespannten Situation sichergestellt werden.

Die ethische Bildung des Arztes soll durch das lebendige Vorbild und die sorgfältige Unterweisung durch den erfahrenen Kliniker und Praktiker erfolgen. Ethische Entscheidungen gehören in den Verantwortungsbereich des Arztes.

freien. Die gewonnenen Erkenntnisse und die technische Entwicklung sollen gezielt nach medizinischen Notwendigkeiten gefördert und eingesetzt werden. Dazu braucht es wieder eine fundierte, am ärztlichen Vorbild orientierte medizinische Aus-, Weiter- und Fortbildung und damit eine Abkehr vom Bologna-Prozess.
Naturwissenschaftlich begründete und menschliche Medizin widersprechen sich nicht, sondern gehören zusammen. Die Hippokratische Gesellschaft setzt sich für die Freiheit der Wissenschaft und Forschung ein und dafür, dass die wissenschaftlichen Erkenntnisse verantwortungsbewusst und zum Wohl der Menschen eingesetzt werden.

\section{Freiheit des Arztberufes:}

Die Unabhängigkeit des ärztlichen Berufsstandes ist Voraussetzung für eine optimale medizinische Versorgung der Bevölkerung. Dazu müssen die Therapiefreiheit, die Fortbildungsfreiheit und die Handels- und Gewerbefreiheit gewährleistet werden. Die Hippokratische Gesellschaft wendet sich sowohl gegen eine sozialistische Staatsmedizin als auch gegen eine Auslieferung des Gesundheitswesens an einen globalisierten Markt ohne ethische und nationale Bindung.

\section{Verantwortung für das Gemeinwesen:}

Die Tätigkeit des Arztes findet nicht nur im Rahmen der ArztPatient-Beziehung, sondern auch im gesellschaftlichen Umfeld statt. Entsprechend soll der Arzt soziale Verantwortung für das Gemeinwohl wahrnehmen. Hierzu gehört auch der Einsatz für die Gesundheitsvorsorge und für gesellschaftspolitische Belange. Für den verantwortungsbewussten Arzt ist die Ausbildung der nachkommenden Medizinergeneration eine vornehme Pflicht.

Historisches Bewusstsein:

Die Grundsätze der hippokratischen Ethik haben sich über 2500 Jahre Geschichte bewährt. Die Schrecken zweier Weltkriege und zahlreicher Terrorregime des 20. Jahrhunderts haben gezeigt, dass immer da, wo die hippokratische Lehre in Frage gestellt oder relativiert wurde, letztlich Ideen im Gesundheitswesen Einzug gehalten haben, die gegen das Leben und die Gesundheit gerichtet waren. Als Beispiel dafür seien der Nationalsozialismus in Deutschland und die kommunistischen Diktaturen genommen, wo sich Ärzte nicht mehr in erster Linie dem Patienten verpflichtet fühlten, sondern sich in Abhängigkeit des totalitären Regimes begaben und von ihrem eigentlichen Handlungsethos, den allgemeinen Geboten der Menschlichkeit zu dienen, abrückten.

Die Hippokratische Gesellschaft setzt deshalb alles daran, ähnliche Entwicklungen frühzeitig zu erkennen und ihnen entgegenzuwirken. Sie trägt dazu bei, dass die Ärzte sich weiterhin an den bewährten ethischen Grundlagen orientieren und ihr Handeln daraus ableiten.

Unsere Grundpositionen sind in obenstehender Positionsbestimmung ausformuliert. Weiteres entnehmen Sie unserer Homepage www.hippokrates.ch

\section{Literatur}

1 Mattli J, Knirsch U, Klesse R, Vuilleumier-Koch S. Zur Bedeutung des Hippokratischen Eides in der heutigen Zeit. Schweiz Ärztezeitung. 2016;97(23):854-6.

2 http://hippokrates.ch/topic/sterbehilfe 